[1]

Dipartimento di Elettronica, Informazione e Bioingegneria, Politecnico di Milano, Milan, Italy.

\title{
Wearable technology: role in respiratory health and disease
}

In the future, diagnostic devices will be able to monitor a patient's physiological or biochemical parameters continuously, under natural physiological conditions and in any environment through wearable biomedical sensors. Together with apps that capture and interpret data, and integrated enterprise and cloud data repositories, the networks of wearable devices and body area networks will constitute the healthcare's Internet of Things. In this review, four main areas of interest for respiratory healthcare are described: pulse oximetry, pulmonary ventilation, activity tracking and air quality assessment. Although several issues still need to be solved, smart wearable technologies will provide unique opportunities for the future or personalised respiratory medicine.

@ ERSpublications

Smart wearable technologies provide unique opportunities for assessing and monitoring respiratory function http://ow.ly/BHXY30cEfBI

\section{The Internet of Things in healthcare}

The term "Internet of Things" (IoT) was originally proposed by Kevin Ashton, a British technologist, in 1999, referring to the connection of everyday objects and industrial apparatus to a data network, enabling information collection and processing to manage these objects and create new services. The development of the loT since the early 2000s has been considerable. Initially, the IoT was mostly related to industrial and commercial logistics. Objects and goods started to be tagged by RFID (radio-frequency identification) or by optical tags (e.g. barcodes and QR codes) in order to allow routing, inventorying and loss prevention. The loT has developed into specific vertical-market applications, such as surveillance, security, transport, food safety and document management. As we are currently observing, in our interconnected world, we are living the phenomenon of the ubiquitous positioning and localisation of people, objects and production through connected wearable devices, connected cars, connected homes, connected cities and the so-called industrial Internet or Industry 4.0, i.e. the "smart factory" where machines, people and information systems are interconnected and integrated with manufacturing that is performed through real-time data acquisition and control of a highly automated production process. In the future, we will experience more and more the ability to monitor and control distant objects, through teleoperation and telepresence.

In healthcare, the concepts of "ubiquitous" and "pervasive" human wellbeing monitoring is becoming a reality thanks to important advances in sensors, miniaturised processors, body area networks (BANs) and wireless data transmission technologies allowing the assessment of physical, physiological and biochemical parameters in different environments and without restriction of activity [1].
Cite as: Aliverti A. Wearable technology: role in respiratory health and disease. Breathe 2017; 13: e27-e36. 


\section{Wearable devices}

"Wearable" means whatever a subject can wear, as sweaters, hats, pants, glasses, bras, socks, watches, patches or devices fixed on the belt, without encumbering daily activities or restricting mobility. The concept of wearability is of particular importance in fields like monitoring for healthcare, wellbeing and fitness/sport. Today, there is an overwhelming number of trendy wearable devices ("wearables") but not all of them are capable of measuring or telling us something reliable about our health status. Conversely, there are plenty of sensors that measure physiological parameters but are not in a wearable form. Wearable biomedical sensors are therefore the subset of devices that are able both to measure physiological parameters and to be worn (figure 1).

Very often, wearable technology is based on conventional electronics, either rigid or bendable, powered by conventional batteries. This includes mobile phone peripherals (devices, interfaces or sensors connected to the phone). In other cases, wearable technology is more "disruptive", and includes apparel and textiles with distributed functions, into which electronics are intimately combined. In this case, the development is not obvious because devices have to be washable, stretchable and foldable, and sometimes printable or transparent.

The ultimate wearable device system should be installed once or continuously, it has to have long lasting battery and very low consumption, wirelessly reporting all health-related information, without constraining its user in any way [3-5]. Calibration has to be performed and maintained, minimising the effects of factors like temperature, humidity and motion (if textile electrodes are used) [5]. Despite these critical features, there is a huge development of wearable devices.

\section{Body area networks}

BANs or body sensor networks (BSNs) are systems composed of a network of wearable devices that can be:

- implanted in the body

- placed on the body in fixed positions

- carried by the person in clothes pockets, by hand or in a bag

In BANs, the communication is entirely within, on or in the immediate proximity of a human body. Let us consider, for example, cardiac monitoring. When continuous deployment is needed, sensors embedded in implantable cardioverter defibrillators, injectable cardiac monitors, implantable loop recorders or pacemakers are used. For shorter periods of monitoring (2-4 weeks), wearable stick-on monitoring devices ("patches") worn on the torso to provide continuous and looping ECG recording are used. When intermittent symptomtriggered monitoring, in the presence of the ability of the subject to activate or initiate recording, touchactivated electrodes configured for smartphones can be used.

In a typical architecture of a patient monitoring system using wearable sensors (figure 2), on-body

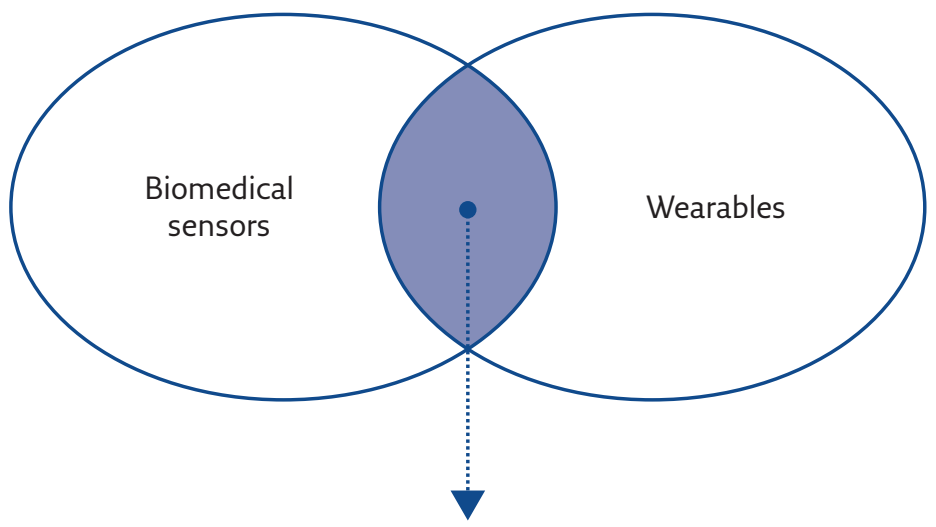

Wearable biomedical sensors

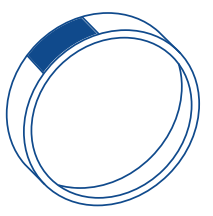

Activity trackers

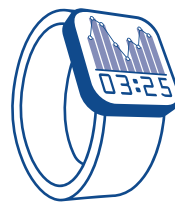

Smart watches

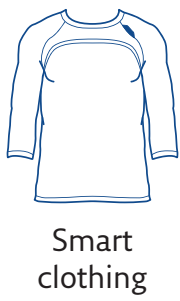

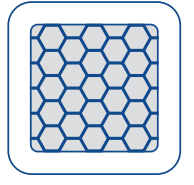

Patches/ tattoos



Ingestibles/ smart implants

Figure 1 Wearable biomedical sensors. Reproduced and modified from [2] with permission from the publisher. 


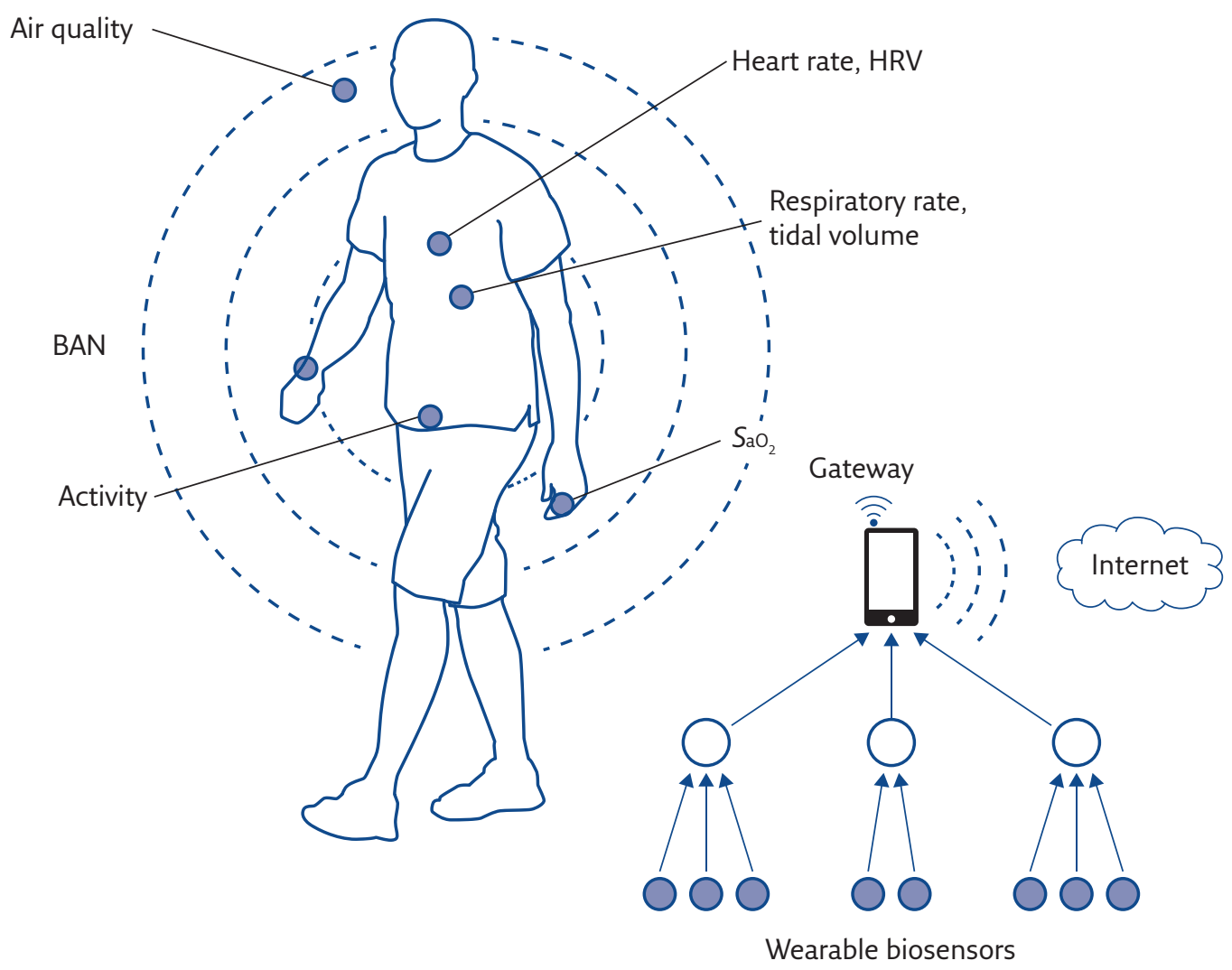

Figure 2 Architecture of a patient monitoring system using wearable sensors. HRV: heart rate variability; $\mathrm{SaO}_{2}$ : arterial oxygen saturation.

wearable sensors wirelessly communicate with stationary (wireless local area network) or wearable (mobile phone) gateway devices to relay sensor data to remote locations. The measurements can be either stored locally on a monitoring wearable device for later transmission or directly transmitted (e.g. over the public phone network) to a medical centre where patient's data can be accessed online using the Internet independently of the patient's location.

Reducing battery consumption through power scavenging (or "energy harvesting") from on-body sources (e.g. motion, vibrations and temperature) or from the environment (e.g. light and temperature) is a possible solution to enhance battery life, particularly for implantable sensors. The development of low-power measurement and transmission systems is key to the successful development of wireless BSN systems [6].

In the last few years, the arrival of Google, Apple, Samsung, Sony, Nike, Philips and others large companies in the field of wearable technology, particularly smart watches able to connect to the Internet, represents the tipping point in terms of accelerating wearables into the mainstream, since these companies are entities with substantial technical and financial resources. Taken together with apps that capture and interpret data, and integrated enterprise and cloud data repositories, the networks of wearable devices and BANs form the foundation of the healthcare loT.

\section{Wearable technology in healthcare: what is it for?}

In healthcare, the majority of diagnostic tools offer information that is essentially a "snapshot in time". The next great challenge is to monitor a patient's physiological or biochemical parameters continuously, under natural physiological conditions and in any environment. The development of wearable devices and wireless BSNs offers a platform to establish such a personalised health monitoring system, with the purpose of recording individual's chronic (long-term) and acute (short-term) events.

In this context, wearable systems should also be able to provide real-time feedback not only to medical staff and patients. These systems will provide an increasing number of solutions for postoperative rehabilitation, to expedite recovery and for independent living for the elderly. They can be used to monitor and track conditions of patients in cities or rural areas, thereby reducing the workload of healthcare providers, increasing efficiency, reducing costs of healthcare services and improving patients' comfort. Mortality can be reduced with correct instructions provided to the patients at the right time.

Healthy subjects performing recreational and sporting activities can track trends and improvements in performance. In professional 
athletes, wearing sensorised garments or smart textiles could help coaches and athletes to assess performance, physiological parameters and body kinematics, and to study an athlete's response (e.g. a racing car driver) to simulated conditions.

\section{Wearable technology for respiratory assessment}

In the recent past, several wearable devices providing useful information for either assessing and monitoring respiratory function of a patients or providing environmental information that can be of interest for the respiratory system have been introduced. This article will give a short summary of:

- arterial oxygen saturation

- minute ventilation, respiratory rate and breathing pattern

- activity

- air quality

\section{Oxygen saturation}

Pulse oximetry is widely used for respiratory monitoring [7]. Pulse oximeters measure oxygen saturation by illuminating a small portion of human skin at two wavelengths, typically $660 \mathrm{~nm}$ (red) and $940 \mathrm{~nm}$ (infrared), and measuring light absorption, which depends on the levels of oxygenated (oxyhaemoglobin) and deoxygenated blood (reduced haemoglobin). Two approaches are typically used.
In transmission oximetry, the light that passes through the body (e.g. a finger or an earlobe) is analysed. In reflection oximetry, the back-reflected light from the body ("backscattering") is sampled at the two different wavelengths and oxygen saturation is estimated from empirical equations. In wearable pulse oximeters, motion artefact and low perfusion are critical; however, recent advances in signal analysis and reflectance technology are significantly improving accuracy, reliability and ability to automatically distinguish artefacts from true signals by analysing signal waveforms.

Figure 3 shows a schematic diagram of the location on the body of several commercially available wearable devices able to perform pulse oximetry, very often in combination with a set of other measurements. Devices can be worn on the wrist like a watch (e.g. in Oxitone 1000 wrist pulse oximeter, www.oxitone.com; Helo lx, www. helo.life/index_enter.html; Vincense whms, www. vincense.com; Gobe activity monitor (combined with blood pressure, respiration rate and body temperature), https://healbe.com; and Visi Mobile (used for fitness and lifestyle applications, estimates calorie consumption by measurements of a pulse oximeter, an accelerometer and a skin impedance sensor), www.soterawireless.com/ visi-mobile). Devices can also be worn on the head, like the Neuroon device (https://neuroon. com/), which is also able to measure electroencephalography, electro-oculography, motion and temperature, and is therefore particularly useful for sleep analysis. A new, interesting trend is to use earphones combining audio features with



Figure 3 Location on the body of several commercially available wearable devices able to perform pulse oximetry. 
pulse oximetry and step counting (Free Wavz, www. freewavz.com/; and DashPro Bluetooth, www. bragi.com/shop/de_en/the-dash-pro.html). More traditional wearable devices are worn on the earlobe (Lumafit, www.lumafit.com) or finger (iHealth Pulse Oximeter, https://ihealthlabs.eu), while lower limb measurements can be performed on the thigh (Humon Hex, https://humon.io), legs (BSX Insight (used for fitness applications), www.bsxinsight.com) or ankle (Owlet baby monitor (for measuring heart rate, oxygen saturation, skin temperature and sleep quality in infants), http://owletcare.com).

Another interesting trend investigated in recent years is noncontact photoplethysmography (PPG), also called imaging PPG (iPPG) [8]. iPPG is based on a mobile phone or computer camera, and is designed to operate without specialised hardware and without the need to wear any special equipment. An area of skin (face, hand, fingertip or earlobe) is placed in front of the camera lens for several seconds to capture several consecutive image frames. In each image of the region of interest, the raw traces of the red, blue and green channels are then obtained, and the green signal is typically used to analyse the cardiac pulse signal. Using advanced signal processing technology, relative physiological signals such as heart rate, respiratory rate (see below), heart rate variability and oxyhaemoglobin saturation are obtained. The principal advantage of $\mathrm{PPPG}$ is that there is no contact between the patient and the measurement apparatus. Although many issues still need to be solved, iPPG represents a promising and low-cost solution.

\section{Pulmonary ventilation}

Although pulse oximetry is fundamental for monitoring the adequacy of ventilation, measuring arterial haemoglobin saturation alone is not enough and it still suffers from a number of practical drawbacks, including reduced perfusion at the site of measurement, as in hypothermia or shock.

Pulmonary ventilation is the product of respiratory rate (or breathing frequency, or number of breaths per minute) and tidal volume (the volume of air that is inhaled and exhaled with each breath). Monitoring pulmonary ventilation means monitoring these two fundamental parameters, and all the parameters that can be derived once inspiration and expiration are distinguished, namely inspiratory time, expiratory time, duty cycle (i.e. the ratio between inspiratory time and total respiratory cycle time), mean inspiratory flow (i.e. the ratio between inspired volume and inspiratory time) and mean expiratory flow (i.e. the ratio between expired volume and expiratory time).

These parameters are fundamental to assess ventilatory function in a patient. Respiratory rate, for example, represents a vital sign used to monitor the progression of illness and an abnormal respiratory rate is a fundamental prognostic factor and an important marker of serious illness.

Although frequently forgotten, respiratory rate has been shown to be able to predict adverse clinical events, such as cardiac arrest or admission to the intensive care unit [9], and to discriminate between stable patients and patients at risk better than pulse and blood pressure measurements [10]. As many heart and lung diseases, such as pneumonia [11], affect respiratory rate, in the clinical environment, respiratory activity, and in particular breathing rate, should be monitored continuously over time in sensitive cases, such as post-operative patients and patients using mechanical ventilation. Trends in minute ventilation in patients with cardiopulmonary diseases should also be used as predictors of cardiopulmonary decompensation potentially leading to complications or exacerbations [12]. Monitoring ventilation during sleep, in terms of hypopneas and apnoeas (i.e. quantifying the occurrence of periods with low or no ventilation), is a necessity in the screening for sleep disorders for timely diagnosis and treatment.

Although, of course, these parameters are of paramount importance when assessing ventilation in a patient, in most cases, they still require the use of a flowmeter embedded in a mouthpiece or a mask or a tube. Capnography is still widely used in clinical practice to assess patients' respiratory activity. Other common and noninvasive respiratory monitoring systems are respiratory inductive plethysmography (RIP) and piezoresistive belts. However, after hospital discharge, these methods are not suitable for respiratory monitoring at home, during daily activities and sleep. There is, therefore, still a need for noninvasive devices designed for longitudinal monitoring of minute ventilation, or at least respiratory rate, in these conditions. For this reason, in the last decade, many start-up companies have arisen developing sensorised garments and several products are already available on the market, although very few with CE or FDA marking.

One possibility is offered by body surface sensors. The chest wall is defined as all of the parts surrounding the lungs and moving with them during breathing, namely the thoracic (ribcage) and the abdominal wall. Since the original paper by KonNO and MEAD [13], a wide variety of measurement methods have been proposed to allow measurements of either the rib cage or abdominal motion. Calibration methods (i.e. isovolume method, changing posture and natural breathing) have also been proposed in order to estimate volume changes of the single compartments, of the entire chest wall and of the lung from measurements of diameters, circumferences or cross-sectional areas. The validity of these calibration methods is generally limited to the conditions during which the calibration is performed.

Different approaches can be used to detect breathing-related chest wall motion (figure 4). One possibility is to measure linear displacements along 

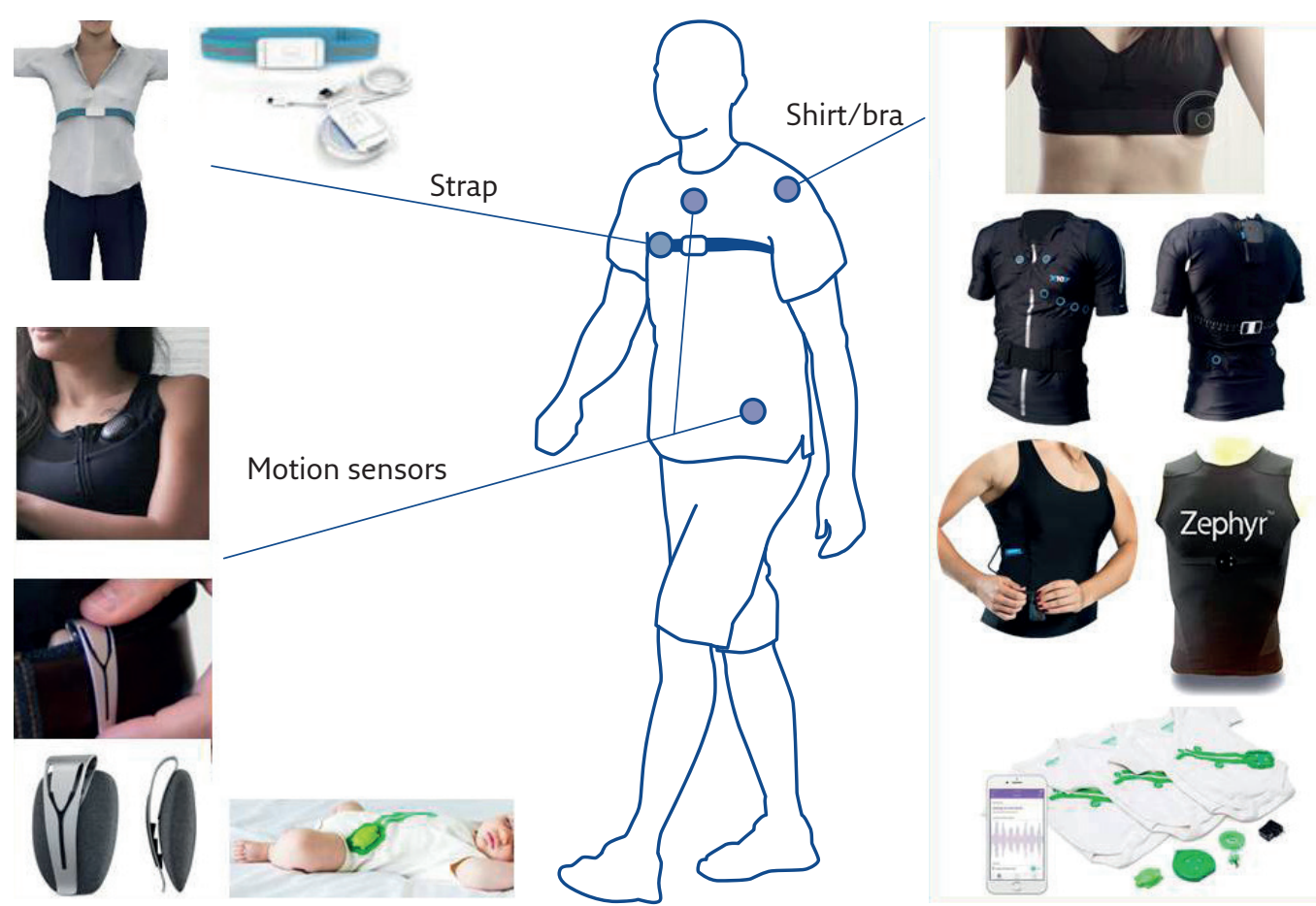

Figure 4 Location on the body of several commercially available wearable devices able to estimate ventilation from chest surface motion.

the anteroposterior axis of the chest. In the past, magnetometers or accelerometers have been used.

More recently, accelerometers worn on the torso have been increasingly used to derive breathing rate by measuring movements of the chest wall [14-17]. This approach, however, is relatively weak with regard to motion artefacts and can be used only under static conditions. For this reason, others have tried to overcome this limitation by using gyroscopes coupled to accelerometers [18] or by adding magnetometers [19]. Nowadays, MicroElectroMechanical System (MEMS) technology uses miniaturised, nine-axis motion tracking devices, combining three-axis accelerometers, three-axis gyroscopes and three-axis magnetometers in the same chip together with a digital processor to process complex sensor fusion algorithms that provide three-dimensional motion reconstruction. The Spire (www.spire.io) and the Vitali Smart Bra\&GEM (https://vitaliwear.com), for example, are wearable devices that measure the user's activity and breathing by motion sensors. Although the manufacturers do not fully disclose the measurement principle, such systems can analyse breathing patterns in terms of breathing frequency and waveform, providing biofeedback to the user.

The MonBaby (www.monbaby.com) is a clip-on device for babies that snaps onto a child's clothing and provides breathing, movement and sleeping position data to a tethered smartphone. MonBaby measurements, based on a MEMS accelerometer, are performed in real-time and transmitted to a smartphone app, which displays the results.
Wearable devices for monitoring babies' breath have great importance, especially for preventing sudden infant death syndrome and the factors that can cause accidental suffocation. Mimo (http:// mimobaby.com) provides another example of a sensorised garment for neonates. Through a smart crib sheet, it measures ventilatory parameters, sleep levels (REM versus non-REM) and movement of the baby on the parents' smartphone or tablet suing an app. The breathing detection system is based on measuring changes in capacitance given two conductive elements (or a series of conductive elements). Conductive elements are positioned on the garment in order to define a fixed spacing and the movement of a body part causes changes in capacitance between the capacitive elements that, once measured, can provide information on the breathing signal. Bluetooth is used to transmit information to a unit positioned near the baby and then upload it to a server where Mimo processes the data for sending to the smartphones app.

Another possibility is to measure changes in chest wall circumference either directly (e.g. using strain gauges) or indirectly (e.g. measuring changes in thoracic impedance). With the former, the variations of the thoracic and abdominal circumferences occurring during breathing are measured through elastic bands that change their electrical resistance with stretching [20] or optical fibres in which light transmission is altered with changes in curvature [21]. Although in the past, strain gauges have been used in some sleep monitoring systems, they have been replaced by RIP. Impedance pneumography represents another 
technique that measures variations in chest circumference by measuring changes in electrical impedance between a pair of ECG electrodes. Electrical impedance tomography instead provides a cross-sectional map of electrical impedance from a set of electrodes in a belt around the chest [22]. An example is offered by Zephyr Performance Systems (www.zephyranywhere.com), now a part of Medtronic, which has developed sensorised garments suitable for several applications, such as defense, first aid and sports. The garment (a compression shirt) and a control unit comprise the system. It monitors heart rate, RR interval, breathing rate, stress level, posture, activity level, peak acceleration, speed, distance and GPS position. The respiration signal is determined through impedance measurements using two electrodes: through them, a drive signal can be applied to the subject, and studying voltage fluctuations, it is possible to obtain the impedance variations in the subject's thoracic cavity due to the respiration. Therefore, the voltage fluctuations may be used to determine the respiratory rate.

Another approach is measuring changes in crosssectional area by RIP, which uses wires incorporated into cloth bands placed around the chest and abdomen. The wires carry an alternating current. Changes in the cross-sectional area enclosed by the band result in proportional changes in self-inductance, causing changes in the voltage measured across the terminal ends of the wires [23]. An example of device based on RIP measurements is that produced by Hexoskin (www.hexoskin.com/ en). This sensorised garment gives the possibility to monitor many parameters. The parameters recorded are heart rate, heart rate variability, heart rate recovery, ECG, breathing rate, tidal volume, minute ventilation and activity (steps, cadence and calories). The sensors for breathing monitoring are textile RIP sensors: electrically conductive loops held closely around a body portion and change of cross-sectional area induces consequent changes in loop inductance. The shirt and the control unit box constitute the device: signals are detected by sensors on the shirt, recorded by a control unit and, through Bluetooth, are transmitted to a smartphone with which is possible to monitor the recorded data. The app interacts with the subject, suggesting increasing running pace or just to recover. The device can be used for $>14 \mathrm{~h}$ if used intensively and for $>150 \mathrm{~h}$ if used only to collect data.

Sensors in textiles can be fabricated by using conductive threads, integrating conductive yarns into the textile structure or printing patterns using conductive ink [24-26]. In all these cases, strain sensors are integrated into the garments, with different manufacturing methods. In the LIFE device (http://x10y.com), which incorporates, in the same shirt, a 12-lead ECG, strain sensors are based on the measurement of the electrical resistance variations of a sensitive segment embedded in the garment. The core of the sensors consists of an elastic strips of conductive ink that is drawn onto a compression shirt or bra by transfer processes or embedded in the textile. AirGo (www.myairgo.com) is a device that measures circumference changes with a silvercoated adjustable strap worn over the lower floating ribs. In this case, a three-axis position sensor, local memory and a Bluetooth device allow data to be collected and either to saved or sent to a remote device.

An alternative approach for monitoring respiratory rate is to derive it from ECG, through a technique called ECG-derived respiration (EDR), or the PPG signal from pulse oximeters, which are both modulated by respiration. EDR is based on the modulating effect that respiration has on the ECG signal. In other words, ECG morphology has small changes during the respiratory cycle caused by movement of the heart position relative to the electrodes and the change in lung volume. Although poorly understood, several different physiological mechanisms cause modulation also of the PPG [27, 28], which result in amplitude modulation, baseline wander and modulation of beat-tobeat intervals (frequency modulation). Recently proposed methods for extracting respiratory rate from segments of PPG signals rely on estimating it from each modulation (the derived respiratory signals) and fusing the three estimates into a final value [27, 29, 30].

Real-time monitoring of respiratory rate, together with heart rate and motion, can be also obtained by a piezoelectric sensor device that can be slipped underneath the patient's mattress (EarlySense, www.earlysense.com). The data are transmitted and shown on a central display station, enabling appropriate action. This can also be applied to sleep monitoring at home.

\section{Physical activity}

Physical activity can be defined as any body movement that uses skeletal muscles and requires more energy than resting [31]. Measuring physical activity is of extreme importance because of its profound impact on health and disease being highly correlated with health outcomes such as death risk, disease incidence, mortality and hospitalisation [32]. It is now common to consider a pulmonary disease like COPD as systemic [33], with muscle wasting, weakness and physical inactivity representing significant extra-pulmonary features [34, 35].

Physical activity can be measured in the field by several different methods [36]. These can be divided into subjective and objective methods, with the former based on direct observations, diaries, activity logs and questionnaires, and the latter measuring physiological parameters, such as metabolic cost, heart rate, body temperature and biomechanical effects, like acceleration, velocity and displacement [37, 38]. 
Advances in sensor technology over recent years have resulted in the availability of the miniaturised accelerometers used in a new generation of activity monitors. These devices are able not only to quantify activities like walking, running, cycling and exercising, but also to recognise the time spent under sedentary conditions and distinguish different postures (sitting, standing and lying down) through the detection of gravity acceleration, i.e. the static force acting on the body.

The modern activity trackers include complex algorithms and software for the automatic recognition of physical activity. "Activity trackers" or "fitness trackers" constitute a subset of consumer wearable devices used for monitoring physical activity- and fitness-related metrics. In recent years, they have become more and more affordable, unobtrusive and reliable. The MoveMonitor (www.mcroberts.nl) and the Actigraph (http:// actigraphcorp.com) are among the most widely studied activity monitors. Hans Van Remoortel and colleagues evaluated the validity of six activity monitors by correlating indirect calorimetry and monitor outputs in 39 patients with COPD, wearing six monitors as well as a portable metabolic system [39]. This is one of the few cases in which the validity of commercially available devices has been established through a comparison against a gold standard. However, this is also an example on how difficult it is to obtain valid results for a significant period of time. In fact, most companies are actively engaged in research and development of the algorithms to determine number of steps, distance travelled and energy expenditure. Therefore, the firmware is frequently updated and the proprietary algorithms, which are very rarely shared, soon become obsolete.

EVENSON et al. [40] recently wrote a systematic review of 22 studies using Fitbit and Jawbone consumer physical activity monitors. They concluded that although not exhaustive, step count is a measure of physical activity. This is highly relevant, as a large number of physical activity monitors (as of November 11, 2016, 414 models (http://fitness-trackers.specout.com) are commercially available and widely used to measure daily step counts. Nonetheless, many consumer physical activity monitors, including Basis Health Tracker, BodyMedia Fit, DirectLife, Fitbit Flex, Fitbit One, Fitbit Zip, Garmin Vivofit, Jawbone UP, MisFit Shine, Nike FuelBand, Polar Loop and Withings Pulse $\mathrm{O} 2$ have accuracies similar to that of research-grade physical activity monitors for measuring steps [39].

Therefore, these devices offer the possibility of researching physical activity in large populations, such as to validate experimental paradigms (e.g. to verify if physical exercise improves quality of life, to determine the effect of exercise intensity on smoking cessation, etc.), to compare physical activity to physical fitness, to investigate associations between behaviour and activity, to measure physical activity to predict health outcomes or to measure physical activity to inform treatment (e.g. chemotherapy) decisions.

Many consumer physical activity monitors use behavioural change techniques to motivate the user, typically through goal-setting, selfmonitoring and feedback [41]. Setting goals and getting feedback on past and current activity is commonly employed, and there are often opportunities for social interactions that can provide support, competition and other motivational strategies.

\section{Air quality}

According to the World Health Organization, air pollution exposure is responsible for 7 million premature deaths every year. The combination of mobile phones with a new generation of wearable devices has made personal environmental monitors able to measure air quality and other environmental data available. The idea is that people wearing such devices could not only check their own surrounding environment but also, when moving around a city, transmit that information to the Internet to create maps of air quality. Several devices are being developed to monitor indoor and outdoor air quality.

TZOA (https://www.tzoa.com) is a device that can be clipped to the clothing or a purse to measure air quality, atmospheric pressure, temperature, humidity, ambient light and ultraviolet (sun) exposure. The wearer (e.g. subjects affected by asthma or parents caring for infants) can use this information to take action (e.g. choosing less polluted roads for outdoor activities or moving into a room with better indoor air quality). In the future, when such devices will be used by a high number of people sending data to a server, these will provide environmental maps. A similar device called AirBeam (www.takingspace.org/ aircasting/airbeam) using light scattering to measure particulate matter, namely PM2.5, has been designed to operate across an open-source platform and to alert the user about low air quality levels. The Lapka Personal Environment Monitor (https://mylapka.com/pem) was proposed in 2012 and includes different sensor modules that can be individually attached to phones and other devices to measure radiation, intensity of electromagnetic fields and humidity.

Another recently proposed solution combining IoT technology with fashion is the French-made WAIR scarf (www.wair.fr/en), which has a multilayer filter that blocks particulates at $\mathrm{PM}>0.1$, including pollen, gasses and bacteria, thus filtering out $99 \%$ of all pollutants. The scarf is also equipped with tiny battery-powered fans to produce fresh air, and has sensors that monitor the air and transmits the data to an app that provides real-time information on the air quality where the user is. 


\section{Ongoing issues}

With the development of ubiquitous connectivity, the lowering costs of wearable sensors and wellness-related devices and increasing smartphone use, the healthcare loT represents a real opportunity and opens important new perspectives in several fields, particularly the monitoring and management of respiratory chronic diseases, and the availability of the "big data" that these devices can provide. However, several problems are still to be solved and major challenges are still to be faced [42].

Firstly, no regulation is present. Data ownership is often not clear. Many companies have agreements that can result in the user to give personal information rights inadvertently to the company, causing distrust and seriously interfering with the integrity of human subject research. Data access for research can be challenging because institutional review boards have to accept data collection procedures proposed by third parties, which have to pass through a negotiation between the company and the researchers. Privacy remains a concern. Informed consent and ethics need to be revisited, considering how they apply to new modes of research. In other words, new models and technologies for informed consent have to be developed. Research methods and data quality are a concern because the data and methods are typically not shared by companies with the research community, and the reliability and validity of the equipment need to be verified.

Last but not least, the vast majority of wearable devices have not been characterised in terms of reliability, measurement accuracy, safety and efficacy. Patients and users have to navigate through thousands of iOS and Android health and fitness mobile applications connected to wearable devices, and determine by themselves the effectiveness and usefulness of these apps and devices. An extremely low number of wearable devices has a CE, FDA or any other mark of conformity, meaning a marking by which a manufacturer indicates the conformity of a device with the applicable requirements set out in the past and future regulatory laws, such as the Regulation of the European Parliament and of the Council on medical devices, adopted by the European Council on April, 2017 [43].

Basically, most wearable devices today available are not medical devices. What is defined as a "medical device"? In the European Union [43], a medical device is "any instrument, apparatus, appliance, software, implant, reagent, material or other article intended by the manufacturer to be used, alone or in combination, for human beings for one or more of the following specific medical purposes:

- diagnosis, prevention, monitoring, prediction, prognosis, treatment or alleviation of disease,

- diagnosis, monitoring, treatment, alleviation of, or compensation for, an injury or disability,

- investigation, replacement or modification of the anatomy or of a physiological or pathological process or state...

"which does not achieve its principal intended action by pharmacological, immunological or metabolic means, in or on the human body, but which may be assisted in its function by such means."

In this context, there is undoubtedly the need for clinical evaluation of most wearable devices, i.e. "a systematic and planned process to continuously generate, collect, analyse and assess the clinical data pertaining to a device in order to verify the safety and performance, including clinical benefits, of the device when used as intended by the manufacturer" of papers published in peer-reviewed scientific journals, of clinically relevant information like clinical follow-up.

Nevertheless, there is a general consensus that if developers, researchers and healthcare providers work together using an approach that consider the requirements of the user, health and wellness services, smart wearable technologies will provide unique opportunities for the future.

\section{Conflict of interest}

Disclosures can be found alongside this article at breathe.ersjournals.com

\section{References}

1. Yang GZ, ed. Body Sensor Networks. London, Springer Verlag, 2006.

2. Ghandi M, Wang T. The Future of Biosensing Wearables. https:// rockhealth.com/reports/the-future-of-biosensing-wearables/

3. Chan M, Estève D, Fourniols J, et al. Smart wearable systems: current status and future challenges. Artif Intell Med 2012; 56: 137-156.

4. Abidoye AP, Azeez NA, Adesina AO, et al. Using wearable sensors for remote healthcare monitoring system. J Sens Technol 2011; 22-28.

5. Marschollek M, Gietzelt M, Schulze M, et al. Wearable sensors in healthcare and sensor-enhanced health information systems: all our tomorrows? Healthc Inform Res 2012; 18: 97-104.
6. Darwish A, Hassanien AE. Wearable and implantable wireless sensor network solutions for healthcare monitoring. Sensors (Basel) 2011; 11: 5561-5595.

7. Jubran A. Pulse oximetry. Crit Care 2015; 19: 272.

8. Liu $\mathrm{H}$, Wang $\mathrm{Y}$, Wang $\mathrm{L}$. A review of non-contact, lowcost physiological information measurement based on photoplethysmographic imaging. Conf Proc IEEE Eng Med Biol Soc 2012; 2012: 2088-2091

9. Fieselmann JF, Hendryx MS, Helms CM, et al. Respiratory rate predicts cardiopulmonary arrest for internal medicine inpatients. J Gen Intern Med 1993; 8: 354-360.

10. Cretikos MA, Bellomo R, Hillman K, et al. Respiratory rate: the neglected vital sign. Med J Aust 2008; 188: 657-659. 
11. Strauss R, Ewig S, Richter K, et al. The prognostic significance of respiratory rate in patients with pneumonia a retrospective analysis of data from 705928 hospitalized patients in Germany from 2010-2012. Dtsch Arzteb/ Int 2014 111: 503-508.

12. Churpek MM, Yuen TC, Park SY, et al. Derivation of a cardiac arrest prediction model using ward vital signs. Crit Care Med 2012; 40: 2102-2108.

13. Konno K, Mead J. Measurement of the separate volume changes of rib cage and abdomen during breathing. J Appl Physiol 1967; 22: 407-422.

14. Hung PD, Bonnet S, Guillemaud R, et al. Estimation of respiratory waveform using an accelerometer. In: 5 th IEEE International Symposium on Biomedical Imaging: From Nano to Macro, 2008. New York, IEEE, 2008; pp. 1493-1496.

15. Bates A, Ling MJ, Mann J, et al. Respiratory rate and flow waveform estimation from tri-axial accelerometer data. In: 2010 International Conference on Body Sensor Networks (BSN). New York, IEEE, 2010; pp. 144-150.

16. Liu G, Guo YW, Zhu QS, et al. Estimation of respiration rate from three-dimensional acceleration data based on body sensor network. Telemed J E Health 2011; 17: 705-711.

17. Mann J, Rabinovich R, Bates A, et al. Simultaneous Activity and Respiratory Monitoring Using an Accelerometer. In: 2011 International Conference on Body Sensor Networks (BSN). New York, IEEE, 2011; pp. 139-143.

18. Yoon J-W, Noh Y-S, Kwon Y-S, et al. Improvement of dynamic respiration monitoring through sensor fusion of accelerometer and gyro-sensor. J Electr Eng Technol 2014; 9 : 334-343.

19. Gollee H, Chen W. Real-time detection of respiratory activity using an inertial measurement unit. In: Engineering in Medicine and Biology Society, 2007. EMBS 2007. 29th Annual International Conference of the IEEE. New York, IEEE, 2007; pp. 2230-2233.

20. Malmberg LP, Seppä VP, Kotaniemi-Syrjänen A, et al. Measurement of tidal breathing flows in infants using impedance pneumography. Eur RespirJ 2017; 49: 1600926.

21. Allsop T, Earthrowl-Gould T, Webb DJ, et al. Embedded progressive-three-layered fibre long-period gratings for respiratory monitoring. J Biomed Opt 2003; 8: 552-558.

22. Frerichs I. Electrical impedance tomography (EIT) in applications related to lung and ventilation: a review of experimental and clinical activities. Physiol Meas 2000; 21: R1-R21.

23. Sackner J, Nixon A, Davis B, et al. Non-invasive measurement of ventilation during exercise using a respiratory inductive plethysmograph. I. Am Rev Respir Dis 1980; 122: 867-871.

24. Carvalho H, Catarino AP, Rocha A, et al. Health monitoring using textile sensors and electrodes: an overview and integration of technologies. In: Proc. Med. Measurements Appl. Int. Symp., Jun. 2014. New York, IEEE, 2014; pp. 1-6.

25. Stoppa M, Chiolerio A. Wearable electronics and smart textiles: a critical review. Sensors (Basel) 2014; 14: 11957-11992.

26. Paradiso R, Pacelli M. Textile electrodes and integrated smart textile for reliable biomonitoring. Conf Proc IEEE Eng Med Biol Soc 2011; 3274-3277.

27. Karlen W, Raman S, Ansermino JM, et al. Multiparameter respiratory rate estimation from the photoplethysmogram. IEEE Trans Biomed Eng 2013; 60: 1946-1953.

28. Tarassenko L, Villarroel M, Guazzi A, et al. Non-contact video-based vital sign monitoring using ambient light and auto-regressive models. Physiol Meas 2014; 35: 807-831.

29. Orphanidou C, Fleming S, Shah SA, et al. Data fusion for estimating respiratory rate from a single-lead ECG. Biomed Signal Process Control 2013; 8: 98-105.

30. Zhu T, Pimentel MA, Clifford GD, et al. Bayesian fusion of algorithms for the robust estimation of respiratory rate from the photoplethysmogram. Conf Proc IEEE Eng Med Biol Soc 2015; 2015: 6138-6141.

31. Caspersen CJ, Powell KE, Christenson GM. Physical activity, exercise, and physical fitness: definitions and distinctions for health-related research. Public Health Rep 1985; 100: 126-131

32. Decramer M, Rennard S, Troosters $T$, et al. COPD as a lung disease with systemic consequences-clinical impact, mechanisms, and potential for early intervention. COPD 2008; 5: 235-256

33. Fabbri LM, Rabe KF. From COPD to chronic systemic inflammatory syndrome? Lancet 2007; 370: 797-799.

34. Waschki B, Kirsten A, Holz O, et al. Physical activity is the strongest predictor of all-cause mortality in patients with COPD: a prospective cohort study. Chest 2011; 140: 331-342.

35. Garcia-Aymerich J, Lange P, Benet $M$, et al. Regular physical activity reduces hospital admission and mortality in chronic obstructive pulmonary disease: a population based cohort study. Thorax 2006; 61: 772-778.

36. Lamonte MJ, Ainsworth BE. Quantifying energy expenditure and physical activity in the context of dose response. Med Sci Sports Exerc 2001; 33: S370-S378.

37. Montoye HJ, Kempen HCG, Saris WHM, et al. Measuring Physical Activity and Energy Expenditure. Chicago, Human Kinetics, 1996

38. Bouten CVC, Koekkoek KTM, Verduin M, et al. A triaxial accelerometer and portable data processing unit for the assessment of daily physical activity. IEEE Trans Biomed Eng 1997; 44: 136-147.

39. Wright SP, Hall Brown TS, Collier SR, et al. How consumer physical activity monitors could transform human physiology research. Am J Physiol Regul Integr Comp Physiol 2017; 312: R358-R367.

40. Evenson KR, Goto MM, Furberg RD. Systematic review of the validity and reliability of consumer-wearable activity trackers. Int J Behav Nutr Phys Act 2015; 12: 159.

41. Lyons EJ, Lewis ZH, Mayrsohn BG, et al. Behavior change techniques implemented in electronic lifestyle activity monitors: a systematic content analysis. J Med Internet Res 2014; 16: e192.

42. Bietz MJ, Bloss CS, Calvert S, et al. Opportunities and challenges in the use of personal health data for health research. J Am Med Inform Assoc 2016; 23: e42-e48.

43. European Parliament, Council of the European Union. Regulation (EU) 2017/745 of the European Parliament and of the Council of 5 April 2017 on medical devices, amending Directive 2001/83/EC, Regulation (EC) No 178/2002 and Regulation (EC) No 1223/2009 and repealing Council Directives 90/385/EEC and 93/42/EEC. Off Eur Union 2017; L117: 1-175. 